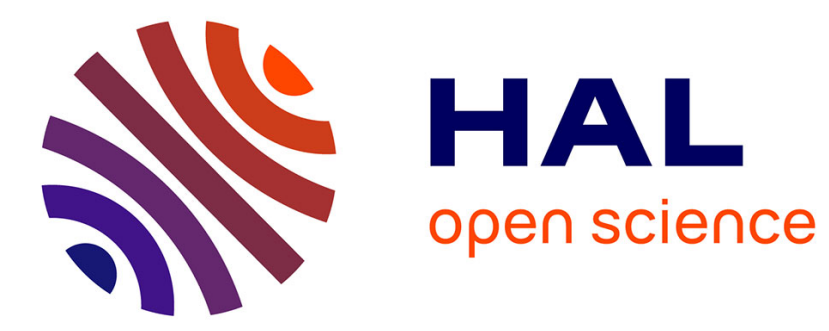

\title{
Sport d'élite et sport pour tous au regard du handicap Jean-Pierre Garel
}

\section{To cite this version:}

Jean-Pierre Garel. Sport d'élite et sport pour tous au regard du handicap. Reliance, 2005, 4, pp.33-44. hal-01936346

\section{HAL Id: hal-01936346 \\ https://hal-inshea.archives-ouvertes.fr/hal-01936346}

Submitted on 7 Dec 2018

HAL is a multi-disciplinary open access archive for the deposit and dissemination of scientific research documents, whether they are published or not. The documents may come from teaching and research institutions in France or abroad, or from public or private research centers.
L'archive ouverte pluridisciplinaire HAL, est destinée au dépôt et à la diffusion de documents scientifiques de niveau recherche, publiés ou non, émanant des établissements d'enseignement et de recherche français ou étrangers, des laboratoires publics ou privés. 


\title{
Sport d'élite et sport pour tous au regard du handicap
}

\author{
Jean-Pierre Garel \\ Cnefei Ccentre national d'études et de formation pour l'enfance inadaptée) \\ Laboratoire Relacs (Recherche littorale en activités corporelles et sportives), Ulco
}

Accéder aux mêmes pratiques sociales que les autres est un droit que les personnes en situation de handicap demandent à pouvoir exercer. Mais une pratique sociale n'est pas bonne en soi. Elle ne mérite d'être promue qu'au regard des valeurs qu'elle véhicule, parfois hâtivement avancées, et donc de la réalité dans laquelle s'incarnent ces valeurs. Le sport n'est pas exempt d'une vigilance, éthique et épistémologique, soucieuse d'une lucidité accrue pour orienter l'action de l'éducateur et du politique.

Si la pratique modérée d'une activité sportive, entendue dans un sens large, est unanimement considérée comme positive, notamment en termes de santé, il n'en est pas de même pour le sport de haut niveau, ou sport d'élite, et même, de façon plus générale, pour le sport de compétition. Bien que souvent paré des plus grandes vertus, le sport doit être l'objet d'un regard sans complaisance, porté ici dans l'intention de pouvoir apporter quelques éléments de réflexion à la question suivante : quelles peuvent être les pratiques sportives des personnes en situation de handicap, du niveau le plus modeste au plus élevé ? On se limitera à décliner cette question en quatre points : en dehors de la compétition, n'y a t'il pas d'autres formes de pratique sportive qui ont émergé ces dernières années et auxquelles des personnes en situation de handicap peuvent ou pourraient accéder ? La référence au sport de compétition, en général, et au sport de haut niveau en particulier, fonde-t-elle la pratique sportive de façon satisfaisante ? Les motivations qui conduisent à s'engager dans une pratique sportive se réduisent-elles au dépassement de soi pour vaincre l'autre ? L'EPS n'a t'elle pas vocation à permettre l'accès de tous aux pratiques convenant à chacun ?

\section{L'évolution des pratiques sportives}

Identifier les formes de pratiques sportives accessibles aux personnes en situation de handicap appelle un détour sur l'évolution de ces pratiques, pour la population française en général, et un préalable sur ce que nous entendrons ici par sport. Tâche moins évidente qu'il n'y paraît, car bien que tout le monde en parle, les meilleurs experts ne parviennent pas à le définir avec précision'. Le sport est souvent présenté comme étant une activité motrice codifiée, donnant lieu à des compétitions, et institutionnalisée. Cette définition vaut pour le sport de haut niveau et une partie du sport dit «de masse ». Elle met en évidence les contraintes auxquelles les sportifs sont confrontés: les règles imposées par l'institution (fédérations, clubs), l'organisation de l'activité selon un calendrier précis et dans des espaces structurés (stades, piscines, gymnase...), et un entraînement rationalisé, nécessaire pour vaincre l'adversaire et

C. Pociello, La culture sportive : pratiques, mythes, représentations, Encyclopaedia Universalis. 
s'engager dans un processus de progrès qui repousse toujours plus loin les limites de la performance. La devise olympique «citius, altius, fortius» symbolise cette recherche continue du dépassement de soi.

Pour beaucoup d'auteurs, le sport est défini par ces seules caractéristiques². Ce qui a le mérite de la clarté mais l'inconvénient d'exclure des activités dont les pratiquants ou leurs spectateurs ont le sentiment qu'elles sont de nature sportive : activités orientées vers la forme physique, les loisirs, l'aventure extrême... C'est pourquoi plusieurs enquêtes s'appuient sur une conception moins restrictive du sport, que nous retiendrons ici pour mieux rendre compte de la diversité des pratiques.

L'enquête publiée en 2002 sur les pratiques sportives en France, par l'Insep (Institut national supérieur de l'éducation physique) et le ministère de la jeunesse et des sports, évalue à 36 millions le nombre de pratiquants de 15 à 75 ans, mais 10 millions seulement sont adhérents d'un club ou d'une association. Les enquêteurs ont en effet pris en compte des pratiquants qui ne sont pas des sportifs au sens institutionnel: des millions de marcheurs, de nageurs, d'adeptes du footing, des sports de glisse sur terre, eau, neige, de la gymnastique d'entretien, etc. Désormais donc, ces pratiques l'emportent largement sur le sport de compétition, en club, dont le sport de haut niveau n'est qu'un aspect concernant une minorité de personnes.

Il existe un lien entre la désaffection des clubs et l'âge. La pratique sportive des personnes âgées de plus de 55 ans augmente et s'effectue peu dans un cadre structuré, mais la tendance s'amorce très tôt, puisque l'on note que, dès l'adolescence, la majorité des activités encadrées par les adultes perdent de leur attrait ${ }^{3}$.

Parmi les activités en vogue, plus ou moins affranchies des contraintes du sport « traditionnel », beaucoup sont apparues en France à partir des années 1970. Certaines étaient tout à fait nouvelles (planche à voile, planche à roulettes, vol libre...), tandis que d'autres renouvelaient des pratiques anciennes (escalade libre, course libre, gymnastique douce...), en profitant parfois de l'évolution du matériel (le roller à la place du patin à roulettes). Ces nouveaux sportifs investissent des lieux qui ne sont pas a priori dédiés au sport: espaces de détente (plages, jardins...), espaces publics urbains (rues, parking...), piétonniers et cyclistes (allées, pistes cyclables), naturels (bois, parcs...).

Concernant les pratiques des personnes en situation de handicap, on peut se référer à des témoignages recueillis notamment auprès d'enseignants d'EPS, de responsables des activités physiques et sportives au sein de collectivités territoriales, et de prestataires d'activités sportives. Tous convergent vers un même constat: les activités qui n'appellent pas de compétition interindividuelle font l'objet d'un intérêt croissant, en particulier celles qui sont dites «de pleine nature ». Patrice Radiguet en témoigne : « À 42 ans, et avec une acuité visuelle de moins de $1 / 10^{\mathrm{m} n}$, je pratique, ou ai pratiqué, la spéléologie et la plongée spéléologique en siphon, l'escalade, le ski de descente et de fond, la randonnée en montagne,

${ }^{2}$ Cf. P. Parlebas, Contribution à un lexique commenté en science de l'action motrice, Insep, 1981.

${ }^{3} \mathrm{M}$. Choquet, Jeunes et pratiques sportives. L'activité sportive à l'adolescence. Les troubles et conduites associées, Rapport au ministère de la Jeunesse et des Sports, Injep, 2001. p. 59. Consultable sur le site http://www.injep.fr/publication/docu/societe.html 
le judo, le canoë, le VTT de trial, de descente et de promenade, le pilotage d'avions de tourisme et l'initiation à la voltige aérienne... »4.

Les progrès technologiques permettent aujourd'hui de disposer d'un matériel techniquement plus accessible (malheureusement pas au niveau financier) dont profitent les personnes en situation de handicap pour leurs loisirs : deltaplane, char à voile et dérivés (le blokart, nouveau concept de «kart à voile»), hand-bike, fauteuil roulant manuel tout terrain, voire flottant pour les activités du bord de mer, catamaran accessibles, ski nautique sur hydrofoil, tir à l'arc pour les aveugles, matériel adapté pour skier, y compris en position assise, « joëlette » pour permettre à des personnes ayant des problèmes de locomotion de participer à des randonnées avec des valides. Même le pilotage des avions, planeurs et autres aéronefs est devenu accessible. Fondée en 1999 par Patrice Radiguet, l'Association Européenne des Pilotes Handicapés Visuels, qui s'intitule aussi «Les mirauds volants », met en exergue cette citation de Marcel Pagnol : «Tout le monde savait que c'était impossible. Il est venu un imbécile qui ne le savait pas et qui l'a fait ».

À côté de l'engouement pour les activités en milieu naturel, on mentionnera la progression des activités physiques artistiques, qui ne relèvent pas du sport à proprement parler mais qui font partie des contenus d'enseignement de l'EPS. Dans le domaine amateur, mais également au sein de troupes professionnelles, participent de plus en plus de personnes autistes, ou porteuses d'une déficience intellectuelle, motrice ou sensorielle.

Demeurent les activités sportives pratiquées dans le cadre des fédérations, spécifiques ou non, avec une évolution notable et souhaitable: le nombre de clubs ordinaires accueillant des sportifs « handicapés » augmente régulièrement. Les conventions avec les fédérations du sport scolaire favorisent également des pratiques mixtes, qui regroupent des jeunes en situation de handicap et d'autres.

Pour clore cette première partie, on soulignera que promouvoir l'intégration par le sport nécessite de prendre en compte l'évolution des pratiques et de la demande sociale, sans se focaliser sur le sport de haut niveau, qui, par définition, ne concerne qu'une élite, ni même sur le sport de compétition dit « de masse », qui exclut une partie de la population lorsqu'elle n'est pas assez performante pour être accueillie ou qu'elle préfère des activités d'un autre type. La référence au sport de compétition mérite par ailleurs de considérer les discussions dont il est l'objet.

\footnotetext{
${ }^{4} \mathrm{P}$. Radiguet, «Pour le plaisir, tout simplement », Place des activités physiques adaptées pour handicapés visuels dans l'enseignement - une aide à l'intégration -, Actes du IIIème congrès européen du Groupement de Recherche et d'Information Consacré à la Cécité et à l'Amblyopie, Bruxelles, novembre 1997, pp. 77-83.

"Cf. le dossier « Des innovations " loisirs " par et pour les personnes handicapées, revue J'accède, n 16, p14-16, (site internet: www.jaccede.com).
} 


\section{La référence controversée au sport de compétition en général et au sport de haut niveau en particulier}

Quand le baron Pierre de Coubertin écrit que «le sport est le culte volontaire et habituel de l'effort musculaire intensif appuyé sur le désir de progrès et pouvant aller jusqu'au risque $»^{6}$, on peut se demander s'il est adapté à toutes les personnes en situation de handicap. L'interrogation devient plus insistante quand il ajoute: "Le sport doit être pratiqué avec ardeur, je dirai même avec violence. Le sport, ce n'est pas l'exercice physique bon pour tous à condition d'être sage et modéré ; le sport est le plaisir des forts ou de ceux qui veulent le devenir $»$.

Dès lors qu'il est conçu comme une activité compétitive et institutionnalisée, le sport risque de donner lieu à une exaltation de la performance, du progrès continûment recherché et du travail qui y conduit, sans souci excessif des effets négatifs qu'il peut induire. Il y a notamment le spectacle désolant des perdants désespérés, c'est-à-dire tous sauf le vainqueur, leurs mines défaites et leurs larmes, que déplore Albert Jacquard et qu'illustre Laure Manaudou, médaille d'or en natation aux J. O. d'Athènes, lorsqu'elle confie : "Pour moi, ne pas être première est synonyme d'échec. Quand j'étais petite, je pleurais quand je ne gagnais pas, même lorsque $\mathrm{j}$ 'avais pris la deuxième place.» Maintenant elle gagne, car « elle a la tronche pour gagner, elle va au carton », selon son entraîneur (Le Monde, 18/8/2004), qui passe dans le milieu pour imposer à ses nageurs un régime de forçat et dont elle suit scrupuleusement les consignes en vue d'un objectif dominant : «Je voulais être championne olympique, point final ».

Pour acquérir le «killing spirit » tant recherché, des méthodes d'entraînement paramilitaire sont parfois les bienvenues. C'est ainsi que les Springbocks (l'équipe de rugby de l'Afrique du sud) ont eu droit à « un entraînement barbare (...) Plonger dans les eaux glacées d'un lac pour gonfler sous l'eau des ballons de rugby, ramper nus sur du gravier, rester confinés nus dans une fosse pendant des heures », etc. (Le Monde, 5/12/2003). D'après le capitaine de l'équipe, «ce stage a eu des effets positifs au-delà de ce qu'on pouvait imaginer ». Certes, le ministre des sports sud-africain a exprimé sa condamnation totale d'un tel entraînement, qui s'accompagne par ailleurs d'attitudes racistes, mais, dans une logique de la performance à tout prix, le résultat prime sur la santé et l'éthique. Cette primauté, qui infiltre le sport de haut niveau et trop souvent le sport de masse, fait dire à Michel Caillat que « le système sportif n'est pas déterminé par la question " qu'est-ce qui est bon pour l'Homme ?" mais "qu'est-ce qui est bon pour gagner ?" $»^{8}$.

Le dopage s'inscrit dans cette logique. Après la révélation d'une utilisation de produits dopants par Marion Jones avant les jeux olympiques de Sydney, Victor Conte, le patron du laboratoire Balco qui fournissait les produits incriminés, renvoie au système la responsabilité des dérapages : «Les gens me demandent souvent si j'ai honte, si j'ai des remords; la réponse est non. Parce que j'ai vite réalisé que, dans le sport de haute compétition, vous devez

\footnotetext{
${ }^{6}$ Cité par J. Defrance, Sociologie du sport, Paris, La Découverte, collection Repères, p. 97.

${ }^{\prime}$ Ibid.

${ }^{8}$ M. Caillat, «La sociologie critique du sport », in Sport et santé : une question d'équilibre, Actes de

l’Université du Sport, Ufolep-Usep, juillet 2004, à paraitre.
} 
faire ce qu'il faut pour gagner; ce sont les règles du jeu. » (Le Monde, 4/12/2004) Une position identique à celle d'Albert Jacquard, pour qui la «dictature de la compétition » conduit inévitablement au dopage.

L'idée qu'il suffirait de vouloir lutter contre le dopage pour l'éradiquer ne prend pas suffisamment en compte les progrès constants des méthodes de fraude, toujours plus imaginatives et en avance sur les procédures de contrôle. Ainsi une hormone de croissance n'est pas détectable dans l'urine et, selon Gérard Dine, « il existe un décalage permanent entre les vagues de progrès sur l'EPO, par exemple, et les actions de lutte contre le dopage ». Il ajoute : «des produits thérapeutiques, issus de la recherche génétique pour vaincre la myopathie, sont d'ores et déjà testés. Nul doute qu'ils seront utilisés par les sportifs lorsqu'ils seront accessibles, sachant que ces produits leur permettront de supporter une charge d'entraînement considérable, à la frontière de la destruction du muscle (...) Le dopage génétique est l'avenir. On peut par exemple agir sur le gène du récepteur de l'EPO pour qu'il soit davantage sensibilisé à cette substance. Actuellement, les travaux sont en cours (...) Les sportifs sont devenus aujourd'hui des machines programmables, de manière interne $»^{\prime}$.

Les athlètes handisport ne sont pas tous exempts de dopage, avec notamment une technique spécifique, le «boosting », qui consiste, pour ceux ou celles dont l'atteinte physique entraîne l'insensibilité à la douleur de certains membres (les jambes pour les paraplégiques), à s'infliger une blessure dont le «bénéfice » est une sécrétion d'adrénaline accrue.

Le dopage et les conduites addictives qu'il entraîne ne sont qu'une des conséquences de la démesure sportive. Loin d'être des phénomènes exceptionnels, les traumatismes physiques sont nettement plus élevés dans la population de sportifs de haut niveau que dans la population ordinaire. Pour Stéphane Cascua, le sport de haut niveau est destructeur de santé ${ }^{10}$. Les enfants qui sont considérés comme des « espoirs» ne sont pas épargnés, au profit «de considérations de prestige, politiques, économiques et sociales, dont les finalités sont bien différentes de celles de l'initiation sportive du jeune $»^{\prime \prime}$.

Pas plus que la santé physique, la santé morale ne trouve son compte dans des tricheries de tout genre. Dans le tournoi de basket pour personnes présentant un handicap mental, lors des jeux paralympiques de Sydney, l'équipe nationale d'Espagne a été disqualifiée pour avoir introduit volontairement un joueur ne correspondant pas au critère de sélection.

Il ne faut pas réduire les dérives du sport au seul haut niveau. Dans le domaine du sport adapté, des compétitions plus modestes peuvent induire la tentation d'affecter un jeune à une division inférieure à ses capacités intellectuelles ${ }^{12}$. Le dopage est également un phénomène qui déborde le champ du sport d'élite : le service «Écoute dopage », créé en 1999, reçoit plus de 3000 appels par an, dont un cinquième provient d'adolescents. Treize pour cent du total des appels concerne une demande d'aide à la décision pour stopper la prise de produits dopants ${ }^{13}$.

" G. Dine, «Les "voies" du dopage », in Sport et santé : une question d'équilibre, op. cit.

${ }^{10} \mathrm{~S}$. Cascua, Le sport est-il bon pour la santé ?, Paris, Odile Jacob, 2002.

M. Caillat, « La sociologie critique du sport », op. cit.

"2 F. Delorme, «Quel entraînement pour quel sportif ? Limite, pertinence, adaptation », in Handicap mental et sport de haut niveau. Limites et pertinence, Colloque européen de la Fédération Française du Sport Adapté, 2526 novembre 2003, pp. 49-55.

${ }^{13}$ Le bilan 2002 est consultable sur internet : www.santesport.gouv.fr/contenu/Lutte_Anti_Dopage/bilan2002.asp 
Des analyses plus exhaustives, fournies par le rapport de Marie Choquet, directrice de recherche à l'Inserm, sur les pratiques sportives des jeunes de 12 à 19 ans ${ }^{14}$ montrent la corrélation entre une pratique intensive, dont elle situe le seuil à plus de huit heures par semaine, et des plaintes somatiques, l'absentéisme scolaire, les conduites violentes et les comportements délictueux, ainsi que la consommation d'alcool, de médicaments, de drogues illicites.

L'obsession de la performance et de la compétition a pénétré toutes les activités de la société contemporaine, engendrant des pathologies coûteuses, mais plus ou moins profondément selon le domaine considéré. Le sport de haut niveau tend naturellement vers la démesure, tandis que le sport de loisir s'en tient plus facilement éloigné : on peut faire du vélo, de la marche, du roller ou nager sans chercher à se dépasser. Ce qui n'exclut pas que ces activités de loisir puissent être pratiquées dans un esprit de dépassement. Paul Yonnet parle de compétition avec soi-même à propos du défi que se lancent des sportifs à l'occasion de ces activités ${ }^{15}$.

Que la compétition soit structurellement porteuse de bien des travers suffit-il à décider de la bannir totalement? Non, car c'est une catégorie anthropologique du jeu, une des quatre catégories fondamentales distinguées par Roger Caillois et qu'il nomme agôn ${ }^{16}$. Autrement dit, la compétition est bien chevillée à l'imaginaire de l'homme, à des degrés divers et excessivement dans notre société. L'éducateur peut et doit tenter d'en prévenir les effets pervers ou promouvoir des pratiques alternatives, mais cela est une autre question.

Quant au procès intenté au sport de haut niveau, il demande à être nuancé. Il est indéniable que la visibilité, dans les media ou ailleurs, de la réussite des sportifs dits « handicapés » a une fonction symbolique que souligne Jacques Defrance : "La représentation de figures de la compétence au sein de groupes dominés sous différents rapports joue un rôle dans les luttes symboliques visant à briser les stéréotypes négatifs attachés à ces groupes $»^{17}$. Par ailleurs, si le sport est structurellement porteur d'excès dont nous avons décrit quelques aspects, ses effets ne sont pas homogènes. Certains athlètes, comme Diagana, ont la sagesse de ne pas verser dans les dérives du dopage, et certaines disciplines sportives, peu médiatisées et $a$ priori physiquement peu traumatisantes, sont moins sujettes que d'autres aux excès. Enfin, dans le domaine du handicap, le sport d'élite présente des singularités qui le tiennent actuellement à un niveau de dérive très inférieur à celui du monde sportif ordinaire. Le temps hebdomadaire d'entraînement y est notamment bien moindre. Pour Aladji Ba, médaillé olympique en athlétisme, sur 400 mètres, il n'est « que » de 18 heures (Le Monde, 16/9/2004), et les sportifs de haut niveau de la fédération française du sport adapté y consacrent seulement deux à huit heures ${ }^{18}$. Ajoutons qu'une grande partie de ces athlètes porteurs d'un handicap mental ont une pratique sportive complémentaire de l'activité compétitive, dans le cadre d'une association ou non. On est loin du sport de haut niveau des fédérations délégataires.

\footnotetext{
${ }^{14}$ M. Choquet, op. cit.

is P. Yonnet, Huit leçons sur le sport, Paris, Gallimard, 2004, p. 72.

${ }^{16}$ R. Caillois, Les jeux et les hommes, Paris, Gallimard, collection Idées, 1967, p. 48.

"J. Defrance, Sociologie du sport, op. cit., p. 77.

${ }^{18}$ A. Marcellini, R. Compte, Les représentations du sport de haut niveau pour les personnes handicapées mentales, Rapport de recherche pour la fédération Française du Sport adapté, 2004.
} 
Une question se pose : si l'on admet que l'intégration des personnes en situation de handicap exige qu'elles accèdent aux mêmes pratiques sociales que les autres, il est logique de revendiquer leur possibilité d'accéder à un statut de sportif de haut niveau identique à celui dont bénéficient des athlètes des fédérations délégataires. Mais ne risque-t-on pas alors d'aboutir aux mêmes conséquences négatives ? Comment conjuguer la volonté d'être comme les autres et celle de maintenir un espace de pratique protégé des dérives constatées?

\section{Le dépassement de soi, une motivation non exclusiv}

La performance est souvent mise en avant pour caractériser le but du sportif. Elle est valorisée, dans la sphère sportive et au-delà' ${ }^{\prime}$, au point d'occulter parfois les autres significations possibles dont le sujet peut investir son activité. On constate ainsi que la recherche de performance, à travers la compétition avec les autres et/ou avec soi-même, n'est pas la seule motivation du sportif. Pour Christian Bromberger, «la fascination pour le sport s'articule autour de trois pôles : le bien-être, le besoin de sociabilité et l'esprit de compétition ( $L e$ Monde, 2/8/2004).

Dans le rapport de Marie Choquet, on retrouve ces trois dimensions sous une forme ou sous une autre. Les motivations pour le sport exprimées par des jeunes de 12 à 19 ans sont les suivantes : le plaisir 74\%, la rencontre avec des amis 37\%, la santé, qui est une préoccupation croissante avec l'âge, $35 \%$, maigrir 20\%, gagner $19 \%$ chez les garçons et $6 \%$ chez les filles.

Ces différentes motivations ne sont pas exclusives les unes des autres : le plaisir de se retrouver entre amis peut se conjuguer avec le désir de se mesurer à eux. Mais, dans certains cas, le choix de la sociabilité s'opère au détriment du désir d'accroître ses performances et même de pratiquer une activité appréciée. C'est ce que nous avons pu constater lors d'entretiens conduits auprès de collégiens présentant une déficience motrice ou une maladie somatique invalidante, qui participent chaque semaine à une séance d'EPS avec leur classe ainsi qu'à une séance spécifique les regroupant en petit nombre. À la question de savoir quelle séance ils préfèrent, nous avons obtenu des réponses variées. Certains désignent la séance spécifique, souvent mieux adaptée à leurs capacités physiques, tandis que d'autres choisissent celle avec leurs camarades de classe, bien qu'ils n'y pratiquent pas toujours des activités adaptées à leurs singularités et que les activités physiques qu'ils disent préférer soient celles qui leur sont proposées lors du cours spécifique ${ }^{20}$. Interrogés sur les raisons de leur choix, leur réponse est sans équivoque : «Pour être avec les camarades ». Ce qui s'exprime à travers ces préférences, c'est la «motivation d'affiliation », véritable «tropisme social » qui pousse à se retrouver avec d'autres personnes et à se faire des amis ${ }^{21}$.

\footnotetext{
19 B. Heilbrunn (dir.), La performance, une nouvelle idéologie, Paris, La Découverte, 2004.

${ }^{20} \mathrm{~J}$.-P. Garel, «Individualiser pour réunir, l'enseignement de l'EPS devant un paradoxe de l'intégration scolaire », in J. Gateaux-Mennecier, M. C. Courteix, Dossier «marginalisation, intégration », La Nouvelle Revue de l'AIS n $\mathrm{n}^{\circ}$ 8, 1999, pp. 153-165.

${ }^{2 C}$ C. M. Alain, «Les théories sur les motivations sociales », in Robert J. Vallerand et Edgard E. Thill (dir.), Introduction à la psychologie de la motivation, Montréal, Éd. Études Vivantes, 1993, pp. 499-506, ainsi que M. Durand, L'enfant et le sport, Paris, PUF, 1987, p. 38-57.
} 
Cette motivation se retrouve dans des activités sportives extra scolaires. Antony, adolescent porteur d'une myopathie de Becker, aime faire du vélo le dimanche avec des copains. Sa faiblesse musculaire et sa fatigabilité l'obligent à mesurer ses efforts. Alors quand une côte se présente sur la route ? «Eh bien tout le monde met pied à terre » répond Antony... Manifestement, ce n'est pas le souci de la compétition qui réunit ces jeunes.

Gagner n'est pas non plus essentiel dans les activités physiques artistiques. En outre, ces activités ouvrent un espace de création dans lequel peut s'exprimer une gestualité qui est davantage bridée par le cadre réglementaire et les techniques de certains sports traditionnels ${ }^{22}$. Elles offrent une marge de liberté et une plage de réussite originale pour des personnes en situation de handicap, ainsi que l'exprime Florence, adulte présentant une déficience motrice : «Dans le sport, les règles sont très strictes. Dans la danse, on nous donne des conseils. On les respecte selon nos possibilités, tandis que dans le sport tout est codifié. Tu ne peux rien changer. En danse, on peut faire plein de mouvements, les agencer librement (...) Il y a une ouverture sur beaucoup de mouvements possibles $\gg^{23}$.

L'engagement dans des activités de pleine nature peut être également vécue comme une expérience de liberté dénuée de tout désir de compétition avec un autre. Il est l'occasion de se mesurer à soi-même et de jouir des sensations qu'offre le contact avec l'environnement. Lors d'une conférence intitulée «Pour le plaisir, tout simplement », Patrice Radiguet décrit des motivations et des émotions distinctes de ce qu'offre la compétition interindividuelle organisée : «Je n'ai jamais cherché à devenir champion olympique d'aucune catégorie sportive, fut-ce handisport. Ma pratique sportive n'est pas l'occasion d'être plus fort que les autres (...) J'ai seulement eu envie, à un moment de ma vie, d'y goûter pour en éprouver les sensations (...) Ce que je recherche ? C'est seulement de vivre, mais avec le maximum d'intensité, en confrontant mon corps et mon être à des sensations vraies et non aseptisées ; j'ai besoin de mordre à pleines dents au fruit sucré de l'existence (...) Solitaire ou accompagné d'amis valides, j'y ai trouvé mon épanouissement physique et mental $»^{24}$.

Ce qui donne du sens à l'activité sportive de Patrice Radiguet, c'est en partie une dépense de soi intense et gratuite, l'occasion de brûler d'un grand feu intérieur, la « consumation » chère à Georges Bataille. Avec David Lebreton, on pourrait parler de « jubilation »25, et avec Roger Caillois de «paidia», ce «principe commun de divertissement, de turbulence, d'improvisation libre et d'épanouissement, par où se manifeste une certaine fantaisie incontrôlée $»^{26}$.

En fait, avec l'ivresse des sensations et l'exultation se conjugue la satisfaction de surmonter des obstacles, dont Patrice Radiguet entend souligner l'originalité par rapport à ce qui peut être vécu en sport de compétition : «Bien plus bricoleur qu'athlète, il m'importe davantage de répondre au "comment vais-je y arriver?", plutôt qu'à: "parviendrai-je à établir une

22 J.-P. Garel, « Expressions corporelles à l'épreuve d'altérations du corps et des sens », in M. Viallefond, J.-P. Klein, Dossier « Art, pédagogie, thérapie », La Nouvelle revue de l’AIS, n¹8, 2002, pp. 97-106.

${ }^{23}$ B. Bouquet des Chaux, « Danser, un envol par-delà le handicap », revue Marsyas, n 39-40, 1996, p. 94.

${ }^{24}$ P. Radiguet, op. cit.

${ }^{25}$ D. Lebreton, conférence «Sport et loisirs », Situations de handicap : quelles ruptures pour quelles mutations culturelles ? Congrès CRHES-Université Lumière Lyon 2, Lyon, 24-26 nov 2004.

${ }^{26}$ R. Caillois, Les jeux et les hommes, Paris, Gallimard, collection Idées, 1967, p. 48. 
quelconque performance sportive ?" Cet "esprit" n'exclut pourtant pas l'effort sur soi librement consenti et la satisfaction de réussir ce qu'on s'est donné pour but ». On peut voir là l'actualisation du second principe anthropologique organisateur des jeux, inverse du principe de la paidia et en tension avec lui. Roger Caillois le nomme «ludus ». C'est « une somme constamment accrue d'efforts, de patience, d'adresse ou d'ingéniosité $\gg^{27}$ qui tend à discipliner l'exubérance. Il participe d'un désir de se dépasser qui n'est pas étranger au désir d'accomplissement personnel.

Faut-il parler d' «accomplissement» ou de «dépassement» de soi ? Se fondant sur une analyse historique, Isabelle Queval établit une distinction « entre la mesure qu'on peut viser, dans l'exercice physique comme en toute chose, et la démesure qu'appelle une performance exceptionnelle ${ }^{28}$; l'accomplissement étant caractérisé notamment par la mesure et le dépassement par la démesure. L'activité décrite par Patrice Radiguet montre une forme de dépassement satisfaisante pour l'éducateur : on n'est pas là devant une recherche exclusive, obsessionnelle et infinie de résultat et d' " amélioration », qui mettrait notamment en péril l'intégrité physique, mais devant le désir de réussir un jour ce sur quoi l'on butait la veille, de dépasser son niveau antérieur. On peut y voir un aspect de la «motivation d'accomplissement », décrite comme l'aspiration à atteindre le niveau le plus élevé possible, à accomplir quelque chose de difficile, à surmonter des obstacles, à se développer et à accroître la maîtrise de l'environnement ${ }^{29}$. Dans la mesure où il n'est pas synonyme d'enfermement dans une bulle individuelle, le souhait d'accomplissement personnel «avec et pour les autres » constitue pour Paul Ricœur le premier niveau de la vie éthique ${ }^{30}$.

Dans le domaine des activités physiques et sportives, l'EPS peut revendiquer un rôle décisif dans l'accomplissement personnel des jeunes.

\section{L'éducation physique et sportive, propédeutique privilégiée et négligée au sport pour tous}

Entre l'éducation physique et le sport pratiqué en club, le rapport généralement évoqué est de l'ordre de la filiation. On considère en effet que l'EPS permet, par les capacités et les compétences qu'elle développe, d'asseoir les performances sportives à venir, qu'elle peut créer le désir de prolonger l'activité physique hors de l'école, et qu'elle constitue un vivier pour détecter les talents qui trouveront à s'épanouir dans une pratique extra-scolaire.

Pour les enseignants d'EPS, leur discipline et le sport ne se confondent pas, bien que les élèves disent volontiers que tel jour ils « ont sport » ou qu'ils vont voir leur « prof' de sport ».

Certes, l'éducation physique puise ses contenus dans le champ des pratiques sportives, mais pas uniquement: les activités physiques artistiques, comme la danse et les arts du cirque, peuvent constituer par exemple un support de travail intéressant. Et, pour l'enseignant, les

\footnotetext{
"R. Caillois, op.cit.

${ }^{2}$ I. Queval, S'accomplir ou se dépasser. Essai sur le sport contemporain, Paris, Gallimard, 2004, p. 14.

${ }^{29}$ Cf. E. Thill, op. cit., pp. 369-377, ainsi que l'ouvrage de M. Durand, op . cit.

${ }^{30}$ P. Ricœur, La critique et la conviction, Entretien avec François Azouvi et Marc de Launay, Paris, Hachette Littérature, collection Pluriel, 1995, p. 142.
} 
activités retenues, sportives ou non, ne sont pas une fin en soi mais au service d'objectifs éducatifs énoncés dans des textes officiels. Concrètement, il peut consacrer du temps à la réflexion des élèves sur leurs façons de travailler, pour qu'ils acquièrent une meilleure méthode de travail, ou à des échanges avec et entre eux pour réguler la vie de groupe et apprendre à vivre ensemble, «perdant» ainsi du temps dans l'acquisition d'une technique sportive.

L'éducation physique peut favoriser la pratique sportive de tous, notamment parce qu'elle est une discipline d'enseignement a priori obligatoire pour tous les jeunes, y compris, donc, pour ceux qui sont en situation de handicap et les plus faibles d'entre eux, alors que le sport relève du temps libre et d'un choix personnel. Chaque élève peut apprendre en EPS à connaitre ses possibilités physiques, à les améliorer pour une meilleure santé, à les mobiliser pour acquérir des habiletés corporelles et sportives, à en retirer des émotions et du plaisir dans des expériences partagées qui peuvent se prolonger ailleurs et dans la vie d'adulte, dans le cadre d'une association, avec des amis ou en famille. Ce sont là des objectifs qui concourent à la visée d'intégration sociale. Le travail effectué en EPS conduit aussi à des apprentissages techniques qui peuvent avoir un bénéfice utilitaire, concernant par exemple le déplacement dans un environnement aléatoire, pour un jeune aveugle, et l'habileté à manipuler son fauteuil à propulsion manuelle pour celui qui est paraplégique.

Pour que l'éducation physique ait une fonction inclusive, il ne suffit pas d'accueillir en classe ordinaire les enfants et les adolescents en situation de handicap ; encore faut-il s'efforcer de favoriser leur réussite sans se limiter à la socialisation. On ne saurait en effet se satisfaire de l'argument selon lequel leur présence « épanouie » parmi des enfants « ordinaires » signe la réussite de l'inclusion. C'est pourquoi nous accordons de l'importance à leurs apprentissages et aux performances qui en témoignent. "Performance »n'est pas ici à entendre dans le sens d'exploit exceptionnel, mais dans celui que l'on peut retenir de la linguistique, à savoir l'actualisation d'une compétence. En d'autres termes, il s'agit des résultats obtenus par les élèves dans des situations concrètes, évaluables. 
L'évaluation est un bon analyseur de l'adaptation de l'enseignement. Elle questionne la pertinence d'une dialectique complexe de l'exigence et de la tolérance, qui est mobilisée par l'enseignant intervenant auprès d'enfants ou d'adolescents qui présentent une déficience. Évaluer les performances de ces jeunes par rapport à une norme qu'ils ne peuvent atteindre témoigne d'une exigence démesurée qui est source d'échec et de renoncement. C'est pourquoi des épreuves spécifiques, ou des critères d'évaluation particuliers pour des épreuves communes, sont construits pour les épreuves physiques des examens de l'Éducation nationale et doivent l'être, si besoin, durant toute la scolarité. Plus que la référence à une moyenne statistique, c'est avant tout le progrès de chacun par rapport à ses performances antérieures qui doit intéresser l'éducateur. Mais la tolérance qui conduit à individualiser l'évaluation devient un obstacle aux progrès de l'élève si elle dérive vers le laxisme. Si en effet, par un respect des différences mal compris, les élèves sont confrontés à des niveaux d'exigence inférieurs à leurs possibilités, on passe à côté d'un objectif essentiel de l'inclusion dans le domaine scolaire : permettre la réussite optimale de tous dans leurs apprentissages ${ }^{31}$.

Au-delà des progrès qu'elle favorise dans le domaine de la motricité, l'EPS en milieu ordinaire est pour les élèves en situation de handicap une occasion de partager avec d'autres jeunes des activités ludiques, bien qu'elles puissent être l'objet d'un travail. Dans la mesure où l'enseignement de cette discipline ne se focalise pas sur la compétition (ce qui n'exclut pas des rencontres entre des équipes ou des individus pour s'en disputer le gain), il offre un temps de vie commune dont on peut espérer qu'il trouve des prolongements hors de l'école.

Malheureusement, il faut bien constater que l'éducation physique des jeunes « handicapés » tarde à trouver sa place dans nombre d'établissements spécialisés et à l'école ordinaire ${ }^{32}$; d'autant plus que, les enjeux de la réussite scolaire induisant une tension dans les familles et chez les enseignants, il est tentant de dispenser d'EPS pour libérer du temps au profit d'autres apprentissages. Par ailleurs, l'évolution des représentations concernant la pratique des activités physiques et sportives par des personnes en situation de handicap est entravée par une représentation dévalorisée de l'éducation physique, en général. En témoigne l'exclusion de cette discipline du socle commun des indispensables défini par le rapport Thélot et reprise par le ministre de l'Éducation nationale. Comme bien des jeunes présentant une déficience rencontrent des difficultés d'apprentissage dans les disciplines «indispensables », on peut logiquement s'attendre à ce que leur temps de scolarité soit consacré à ces disciplines, et donc à ce qu'ils soient souvent dispensés d'EPS. Sachant qu'une étude américaine, rapportée par le magazine Handitec, montre que la proportion de sujets obèses aux Etats-Unis, parmi les adultes porteurs d'une déficience, est de 24,9\% alors qu'elle est de 15,1\% dans le reste de la population, on peut imaginer une évolution dans le même sens en France ${ }^{33}$. On verra alors un groupe d'experts examiner les conséquences de l'inactivité physique sur la santé et proposer des mesures de remédiation!

\footnotetext{
${ }^{31}$ J.-P. Garel, « Respecter les différences : une exigence ambivalente », in C. Philip, C. Gardou, Dossier

«Éthique, éducation et handicap », La Nouvelle Revue de l'AIS, n 19, 2002, pp. 23-34.

${ }^{32}$ J.-P. Garel, « Débats autour de l'intégration », Revue EP.S, n³01, mai-juin 2003, pp. 69-72.

" «L'obésité infantile, "enjeu majeur de la santé", ne cesse de gagner du terrain ». C'était un titre du journal Le Monde, en pleine page (11-12/4/2004). En France, la proportion d'enfants d'âge scolaire présentant un excès de poids est passée de 3\% en 1965 à 16\% aujourd'hui.
} 
C'est ce qui se passe au Canada. Face au constat que 30 pour cent des enfants canadiens sont considérés trop gras ou obèses, que seulement $54 \%$ des jeunes d'âge scolaire (de 5 à 14 ans) participent à des activités sportives, que l'obésité infantile rend vulnérable à des maladies chroniques, comme le diabète, et à des problèmes cardiovasculaires, et que l'inactivité physique coûte annuellement à l'Ontario 1,8 milliard de dollars en soins de santé directs et indirects, le premier ministre de cette province, M. Dalton McGuinty, vient de décider d'investir 5 millions de dollars par an dans le programme d'action «Vie active 2010 », destiné, selon ses propos, à « renforcer l'éducation et les compétences de tous, à améliorer la santé des gens et à assurer notre prospérité. $\gg^{34}$ Il serait souhaitable que, pour sa part, la France soit sensible à ces problèmes de santé liés à l'inactivité physique et qui touchent prioritairement les plus vulnérables.

\section{Pour conclure}

Par définition, le sport de haut niveau ne concerne qu'une minorité d'individus. Se focaliser sur lui ne permettrait pas de répondre à une question fondamentale : comment favoriser l'accès du plus grand nombre de personnes en situation de handicap à une pratique sportive ? Dans ce but, il convient de se tourner vers des activités plus accessibles et, autant que possible, partageables avec des personnes «ordinaires». Ces activités peuvent être de nature compétitive dans la mesure où elles sont suffisamment adaptées aux capacités des sujets, car la recherche de la victoire exclut fatalement les moins performants. Elles peuvent aussi être dénuées de tout esprit compétition avec autrui et même être pratiquées hors d'un cadre institutionnel, seul, avec des amis ou en famille. C'est donc la variété des activités qu'il est souhaitable de promouvoir, susceptible de répondre à la diversité des capacités et des motivations. En effet, l'engagement dans une activité n'est pas mû pour tous par l'intention de se dépasser dans des compétitions organisées dans un cadre institutionnel. Par ailleurs, les dérives constatées dans les compétitions, notamment dans le domaine du sport de haut niveau dont les media rendent compte, posent question : comment, pour des athlètes « handicapés », concilier le désir légitime de cultiver leur excellence et les maintenir à l'écart de ces dérives ?

Le sport peut permettre à des personnes en situation de handicap et des personnes « ordinaires » de vivre ensemble. Par exemple, une manifestation sportive peut donner lieu à la coexistence d'une pratique des uns et des autres, dans un même lieu et un même temps. On évoque souvent la possibilité d'organiser simultanément les jeux paralympiques et les jeux olympiques. Autre forme d'expérience partagée, les pratiques mixtes. Dans ce cas, les personnes en situation de handicap et les autres ne sont plus à côté, juxtaposées, mais engagées dans une interaction motrice commune : marathon debout ou en fauteuil, basketfauteuil pour tous... La limite de certaines de ces pratiques tient à l'esprit de compétition, qui implique une relative égalité des chances de victoire entre les compétiteurs et qui donc élimine les plus faibles. Pour répondre au mieux aux besoins de chacun, à la diversité des activités devrait donc s'ajouter la diversité des contextes de pratique, d'une pratique «entre soi » à une pratique mixte.

\footnotetext{
${ }^{34}$ Site internet : www.activeliving.ca/French/index.cfm
} 
Quant à l'EPS, outre qu'elle favorise la santé et l'acquisition de capacités, de compétences et de motivations susceptibles de s'exprimer dans des activités sportives hors de l'école, elle offre l'opportunité de pratiques mixtes qui concourent à sa fonction inclusive. Par ce qu'elle s'adresse à tous les enfants dès le début de leur scolarité, elle peut contribuer à entraver les préjugés, à porter sur les personnes différentes un regard qui s'attache moins à ce qui distingue qu'à ce qui réunit. 